\title{
Lapurdum
}

Euskal ikerketen aldizkaria | Revue d'études basques |

Revista de estudios vascos | Basque studies review

19 | 2016

Numéro XIX

\section{Petite leçon de dialectométrie à partir des données du Nouvel Atlas Linguistique de la Basse-Bretagne}

\section{Guylaine Brun-Trigaud and Jean Le Dû}

\section{OpenEdition}

\section{Journals}

Electronic version

URL: https://journals.openedition.org/lapurdum/3246

DOI: 10.4000/lapurdum.3246

ISSN: 1965-0655

\section{Publisher}

IKER

Printed version

Date of publication: 1 January 2016

Number of pages: 133-151

ISBN: 978-2-95534-134-6

ISSN: $1273-3830$

Electronic reference

Guylaine Brun-Trigaud and Jean Le Dû, "Petite leçon de dialectométrie à partir des données du Nouvel Atlas Linguistique de la Basse-Bretagne", Lapurdum [Online], 19 | 2016, Online since 01 January 2021, connection on 03 September 2021. URL: http://journals.openedition.org/lapurdum/3246 ; DOI: https:// doi.org/10.4000/lapurdum.3246

Creative Commons - Attribution - Pas d'Utilisation Commerciale - Pas de Modification 4.0 International - CC BY-NC-ND 4.0 


\section{Petite leçon de}

\section{dialectométrie à partir des données}

\section{du Nouvel Atlas Linguistique de la Basse-Bretagne}

Guylaine Brun-Trigaud

Université de Nice-Sophia-Antipolis

Jean Le Dû

Université de Bretagne Occidentale

On le sait la dialectométrie n'a pas été au cour des recherches de la dialectologie française, alors que le nom lui a été donné par l'un de ceux qui ont largement contribué à l'entreprise des Atlas Linguistiques de la France par régions, nous voulons parler de Jean Séguy. À de rares exceptions près, et Charles Videgain en fait partie, Séguy n'a pas été suivi dans cette voie pourtant prometteuse et c'est à l'étranger que la discipline s'est largement développée ces dernières années. Il ne faut pas cacher que c'est Guylaine Brun-trigaud qui a entraîné Jean Le Dû dans cette aventure à laquelle il n'était pas hostile, mais dont il n'était pas non plus complètement convaincu du bien fondé. Pourtant aujourd'hui il existe des outils commodes et bien pensés qui facilitent grandement la tâche, même si la mécanique en semble encore parfois un peu mystérieuse pour les dialectologues qui n'ont pas toujours des affinités avec les statistiques. Ce sont les péripéties de cette aventure que nous allons raconter ici, en hommage à ceux qui, comme Charles Videgain, croient que la dialectométrie peut apporter une perspective nouvelle à la dialectologie et à la géolinguistique.

\section{Présentation du NALBB}

Jean Le Dû nous donne d'abord quelques détails sur la conception du Nouvel Atlas Linguistique de la Basse-Bretagne dont il est l'auteur : cet atlas comprend 601 cartes et 187 points d'enquête (figure 1) répartis sur la Basse-Bretagne ou Bretagne bretonnante, ensemble de 600 communes couvrant le département du Finistère et la partie occidentale des Côtesd'Armor et du Morbihan. Les enquêtes de terrain, commencées en 1969, se sont étalées sur une trentaine d'années. Latlas devait être le premier d'une série : ces deux premiers volumes, surtout destinés à préciser les limites dialectales les plus représentatives, s'appuient sur un questionnaire portant essentiellement sur des notions simples, connues de tous. Ce qui explique que de nombreuses cartes sont monolexématiques et que la notation phonétique, entièrement faite par Jean Le Dû afin d'éviter les différences d'appréciation des sons, est impressionniste et très attachée à représenter les nuances. Les volumes suivants devaient comprendre un nombre réduit de points choisis en fonction de l'analyse des résultats de cette 
première série d'enquêtes. Ils n'ont pu être réalisés faute de temps et de moyens. le travail d'enquête pour les deux premiers volumes a entièrement été réalisé par l'auteur et plusieurs collaborateurs bénévoles bénéficiant pour certains de frais de déplacement accordés par le GDR 9 du CNRS «Atlas Linguistiques », à la différence d'autres atlas français qui sont l'œuvre de personnels dédiés à plein temps à cette tâche. Les parlers traditionnels sont actuellement en voie d'extinction rapide, et il est heureux qu'un groupe de chercheurs bénévoles ( http:// banque.sonore.breton.free.fr/ ) poursuit - sans aide officielle - un grand travail de collecte cette fois très poussé du point de vue grammatical qui permettra d'élaborer un atlas complet.

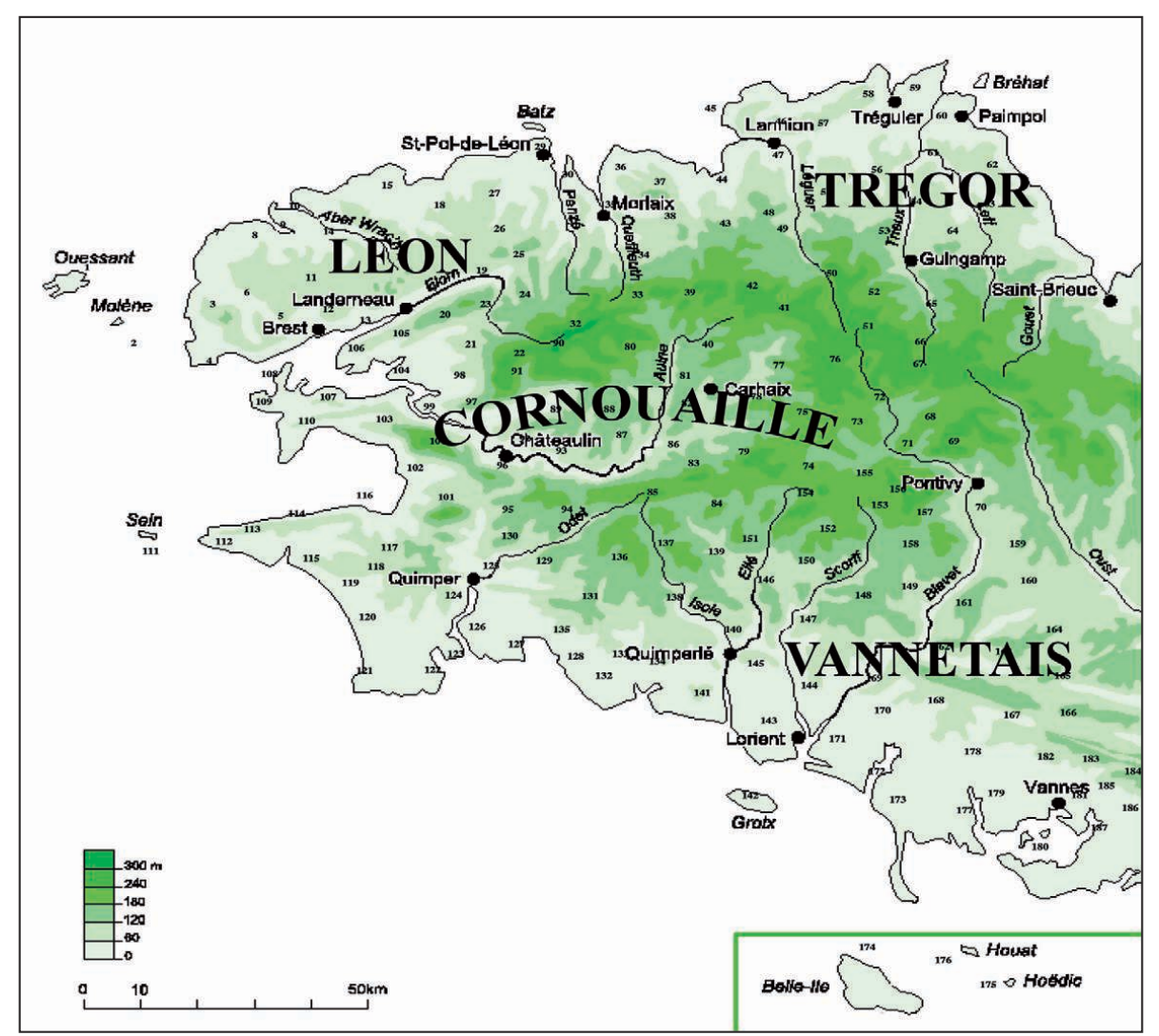

Figure 1 : Bretagne physique et principales divisions dialectales du breton

Les noms qui figurent sur cette carte (Figure 1) permettront au lecteur de s'y retrouver dans les commentaires, et il pourra voir l'importance des rivières dans la répartition des faits (la taille réduite des cartes interprétatives n’a pu permettre de les y reporter).

\section{Quelques mots sur la dialectométrie}

La dialectométrie a pris son essor à Toulouse sous l'égide de Jean Séguy (1971 \& 1973), mais aussi d'Henri Guiter (1973) qui, après la disparition prématurée de Séguy, a produit de nombreux travaux quantitatifs sur les atlas régionaux français. Il s'agissait, dans l'esprit de son créateur, de trouver de l'ordre dans le chaos, d'« élaborer une matière informe et insaisissable et mener les données quantitatives jusqu'à leur formalisation terminale et mathématiquement 
nécessaire » [...] « Qui a pris, dit-il, l'initiative de traiter, au sens informatique du mot, les multitudes de notations micro-phonétiques de l'ALF et de sa descendance ? Il est vrai que l'entreprise est peu engageante. Ne parlons pas de la perspective d'une tâche lourde, longue et assidue. Mais ce qui décourage c'est la dispersion des faits. » 1973: 2-4.

Pourtant bien vite, bénéficiant des débuts de l'informatique, d'autres chercheurs ont poursuivi ce type de travaux. Parmi eux, il faut citer Hans Goebl qui jette rapidement les bases de la dialectométrie classificatoire et surtout de ses représentations cartographiques en utilisant la géométrie de Delaunay et de Voronoi (polygonisation). L'ALF sera d'ailleurs l'un de ses terrains favoris pour mettre en œuvre différentes formes de classifications basées sur la taxation de chacune des cartes étudiées (environ 45\% de l'ensemble des cartes). Les cartes retenues sont utilisées selon leurs propriétés. Si elles sont multi-lemmes, elles forment alors le groupe des cartes lexicales exploitées soit de façon binaire (tous les lemmes ont la même valeur), soit de façon pondérée (selon le principe où les lemmes les plus rares ont plus de poids que les lemmes les plus fréquents (Gewichteter Identitätswert GIW)). En revanche, les uni-lemmes forment le groupe des cartes phonétiques (subdivisées en étude du vocalisme et du consonantisme) où un ou plusieurs éléments de l'étymon du lemme font l'objet d'une typisation à part. Elles composent également, le cas échéant, le groupe des cartes morphosyntaxiques,. Les matrices de données issues de cette taxation sont ensuite traitées pour être converties en matrices de similarité et/ou de distance à l'aide de l'IRI (indice relatif d'identité) qui fait écho à l'IRD (indice relatif de distance) (IRI + IRD = 100), auquel il faut ajouter l'IPI (indice pondéré d'identité). Enfin les résultats de ces données donnent matière à diverses représentations cartographiques basées sur des algorithmes classificatoires : synthèses isoglottiques (cartes à cloisons), cartes de similarité, à rayons, à paramètres, à corrélations et des arbres « dendrogrammes » (cf. notamment Goebl 2012 pour un panorama complet sur l'École de Dialectométrie de Salzbourg, et surtout 2014 qui récapitule de façon très claire l'ensemble des travaux menés sur l'ALF). On peut consulter les données sur http://www. dialectometry.com/index.php.

D'autres écoles dialectométriques verront le jour un peu plus tard, notamment l'École de Groningen autour de John Nerbonne (2001) et Wilbert Heeringa (2004). Ces derniers utilisent les principes de la distance d'édition (algorithme de Levenshtein) : celle-ci permet de mesurer la distance linguistique entre deux chaînes de caractères, distance qui est égale au nombre minimal de caractères qu'il faut supprimer, insérer ou remplacer pour passer d'une chaîne à l'autre. La comparaison se fait donc sur la totalité de la chaîne et s'exprime en valeur de coût ou en pourcentage de similarité.

Dans l'exemple (1), on compare les variantes recueillies pour la notion « chaîne » (en breton chadenn, carte NALBB 306) dans les points 1 (île d'Ouessant) et 103 (Argol) :

$\begin{array}{llllllll}1 & 1 & \int & \text { a: } & \text { d } & \text { e } & \text { n } & \\ 103 & 1 & 3 & \text { a: } & \text { d } & \text { o } & n & \\ \text { coût } & 0 & 1 & 0 & 0 & 1 & 0 & =2 \\ & 66 \% & \text { de similarité } & & & \end{array}$

C'est au sein de l'école de Groningen que Peter Kleiweg a développé en 2004 une plateforme RuG/L04 (http://www.let.rug.nl/kleiweg/L04/) basée sur le langage R (logiciel libre 
de traitement des données et d'analyses statistiques) devenue, depuis 2010, une application en ligne Gabmap ${ }^{1}$ (http://www.gabmap.nl) (Nerbonne 2011).

Enfin, il faut mentionner aussi l'existence d'une autre application en ligne, Diatech (http://eudia.ehu.es/diatech/index/), créée à l'initiative de G. Aurrekoetxea : elle travaille à la fois sur les modèles de l'École de Salzbourg (IRI, IRD, IPI) et de celle Groningen (Levenshtein) et offre les mêmes possibilités cartographiques, mais en se basant seulement sur les modèles de Salzbourg pour la classification².

En ce qui concerne plus particulièrement le breton, Gary German (1991) est le tout premier à avoir appliqué une approche dialectométrique aux données dialectales. Grâce à une analyse de groupe (cluster-analysis) il a divisé le territoire de la Basse-Bretagne en plusieurs zones selon leur proximité linguistique avec le breton de Saint-Yvi (Finistère sud) qu'il a décrit dans sa thèse de doctorat (1984). Plus récemment Denis Costaouec (2012) a montré les corrélations entre distribution de faits dialectaux internes au breton et divers facteurs sociaux, culturels ou économiques autour du village de la Forêt-Fouesnant dont il a décrit le dialecte dans sa thèse (1999). Ces deux études sont basées sur des données tirées de l'Atlas Linguistique de la Basse-Bretagne (ALBB) de Pierre Le Roux (1924-1963) dont les données ont été recueillies entre 1913 et 1920 (87 points d'enquête, 600 cartes).

\section{Choix et présentation de Gabmap}

A la suite de premiers travaux utilisant l'algorithme de Levenshtein, Guylaine BrunTrigaud (2014 et 2015) a proposé à Jean Le Dû de poursuivre l'expérience sur l'ensemble du domaine du NALBB, en utilisant le logiciel Gabmap découvert lors du dernier colloque Methods in Dialectology (Groningen, 2014).

Nous avons d'abord apprécié la facilité d'intégration des données et surtout les multiples outils permettant à la fois de contrôler les données, d'en établir des statistiques et d'en visualiser les résultats. Nous ne les décrirons pas tous ici, nous rendrons compte seulement de ceux que nous avons utilisés. Un tutoriel régulièrement mis à jour ${ }^{3}$, des manuels en anglais et les nombreux articles de Nerbonne et de ses collaborateurs (tous accessibles en ligne $)^{4}$ permettent de se familiariser très vite avec l'application. On trouvera également les avis d'autres utilisateurs notamment dans Snoek 2014, Guerrab 2014 et Kellerhals 2014. Il est à souhaiter que le site permettra la mise en relation de la communauté des utilisateurs grâce à l'affichage des projets en cours, à l'instar de ce qui se passe pour Diatech.

Pour mener notre expérience à bien, nous avons établi trois corpus distincts à partir des 601 cartes du NALBB. Afin de respecter les recommandations en vigueur dans l'univers de la dialectométrie, nous avons soigneusement écarté les données lacunaires et nous avons, par conséquent, décidé d'éliminer le point 69 (Caurel), situé sur la bordure orientale du domaine,

1. Le nom est forgé sur l'anglais gab « bagout, éloquence » et map « carte ».

2. Nous n'avons pas pu utiliser cette application pour des questions matérielles et de temps.

3. Lapplication est en constante évolution grâce à une maintenance informatique suivie.

4. http://urd.let.rug.nl/nerbonne/papers/ 
qui comportait près de $50 \%$ de non-réponses (l'enquête avait été faite auprès de la dernière locutrice native, disparue au moment de l'enquête complémentaire). Nous avons également retenu en priorité les cartes qui comportaient moins de 10\% de lacunes.

Puis nous avons constitué trois groupes dans l'optique de la comparaison : un premier groupe de 153 cartes uni-lemmes comportant seulement les variantes phonétiques d'un seul lemme pour l'étude sur la phonétique, un deuxième groupe de 196 cartes uni- et multi-lemmes (de 1 à 42 lemmes) et un troisième groupe de 142 cartes multi-lemmes (de 2 à 25 lemmes) pour l'étude sur le lexique. Le but était d'éprouver si la présence de cartes uni-lemmes avait ou non une influence sur la classification en lissant les différences.

Dans chacun des groupes, nous n'avons qu'une seule donnée (ou absence de données) dans nos tableaux, à l'intersection lieu/notion. Même si Gabmap gère les réponses multiples, nous n'avons pas testé cette possibilité.

\subsection{Intégration des données}

Guylaine BT qui travaille sur la base de données du Thesaurus Occitan ${ }^{5}$ s'est chargée de cette tâche technique en suivant les indications dispensées sur le site : les données ont donc été intégrées directement à partir d'un tableur, encodées en Unicode (UTF-8 ou UTF-16) sous la forme d'un fichier texte. Quant au fond de carte, il a été facilement généré à partir du positionnement géolocalisé des points d'enquêtes entourés d'un contour dans Google Earth, puis il a été importé dans Gabmap sous forme de fichier KML

Au moment de l'importation des fichiers, il faut choisir le type de données à intégrer : par exemple, pour des données phonétiques, il faut choisir « string data » puis choisir entre «plain» qui comparera chaque caractère individuellement y compris les diacritiques, ou « tokenized » qui comparera les combinaisons de caractères (c'est la solution que nous avons choisie), ou encore, si l'on a des compétences dans le langage Python, il est possible d'importer son propre fichier de caractéristiques (sinon l'application utilise des caractéristiques standards). Pour les données lexicales, il faut choisir « categorical data » et là encore, choisir entre « binary comparison », qui compare les données sans pondération (notre choix) ou "Gewichteter Identitätswert (GIW) » avec pondération (voir plus haut).

Enfin deux autres choix sont possibles : «numeric data » et « difference data » qui sont, notamment, utilisables si l'on a déjà produit des calculs statistiques.

\subsection{Les outils de contrôle}

Lorsque les données sont intégrées, plusieurs outils permettent de les contrôler et de les visualiser avant toute forme d'analyse statistique : le module «Index/Places » permet de vérifier que le fond de carte créé à l'aide de la polygonisation classique correspond bien aux attentes. Le réseau du NALBB étant assez régulier, il n'offre pas les distorsions de surfaces que l'on remarque parfois sur certaines aires polygonisées...

5. http://thesaurus.unice.fr/index.html 
Le module «Index/Items » donne la liste des cartes chargées et permet de visualiser le taux de remplissage, soit pour l'ensemble des cartes, ce qui permet de contrôler facilement que tous les points sont renseignés, soit individuellement pour chacune des cartes. Il faut dire, et ceci est valable pour l'ensemble du site, que toutes les cartes générées sont téléchargeables sous trois formats : eps, png et pdf (Guylaine recommande ce dernier format pour ceux qui seraient tentés par des retouches dans d'autres logiciels ... et a un petit regret, l'absence de légende automatique).

Toujours en ce qui concerne la vérification, le module « Data Inspection » propose deux outils : le premier, "Data Overview », permet, dans le cas des données phonétiques (string data), d'avoir la liste complète des caractères et des signes diacritiques utilisés (bien utile pour vérifier les erreurs de saisie inhérent à ce genre d'entreprise ...), avec une particularité que Jean a trouvé très intéressante, qui est celle de pouvoir cartographier la répartition de tel ou tel phonème (Figure 2) ou combinaison de phonèmes quelle que soit sa place dans la chaîne.
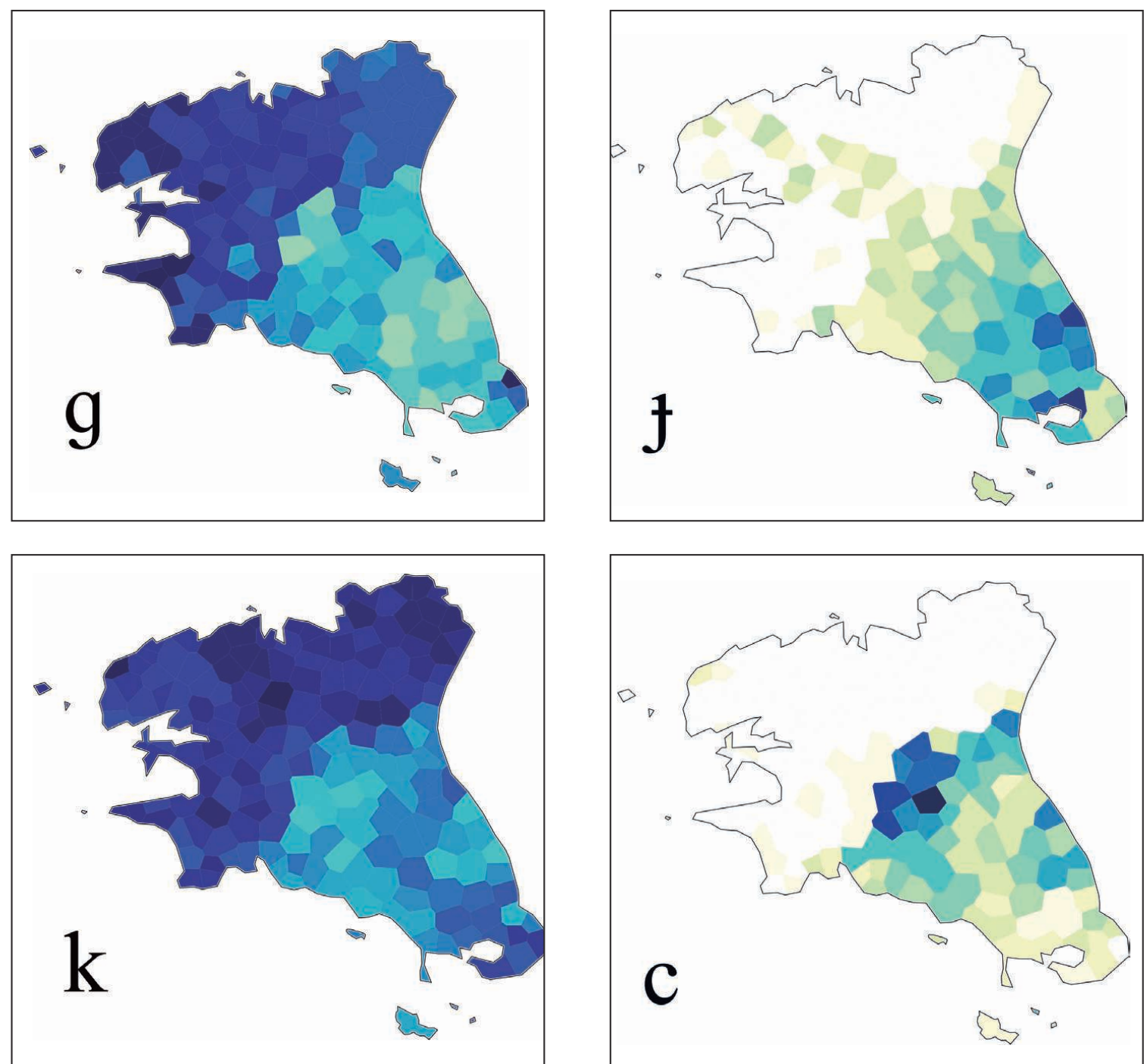

Figure 2 : répartition des phonèmes $\mathrm{g}, \mathrm{f}, \mathrm{k}$ et $\mathrm{c}$ 
Falc'hun a montré (voir figure 3) que la Basse-Bretagne était divisée en deux parties selon un axe NO/SE en ce qui concerne la palatalisation des consonnes : cette division ressort très bien ici. Selon son interprétation, cette opposition serait due à une présence plus grande au NO d'immigrants venus de l'île de Bretagne, comme le montre le plus grand nombre de noms de paroisses en Plou- d'origine insulaire, alors que l'autre partie serait restée plus proche du gaulois armoricain.

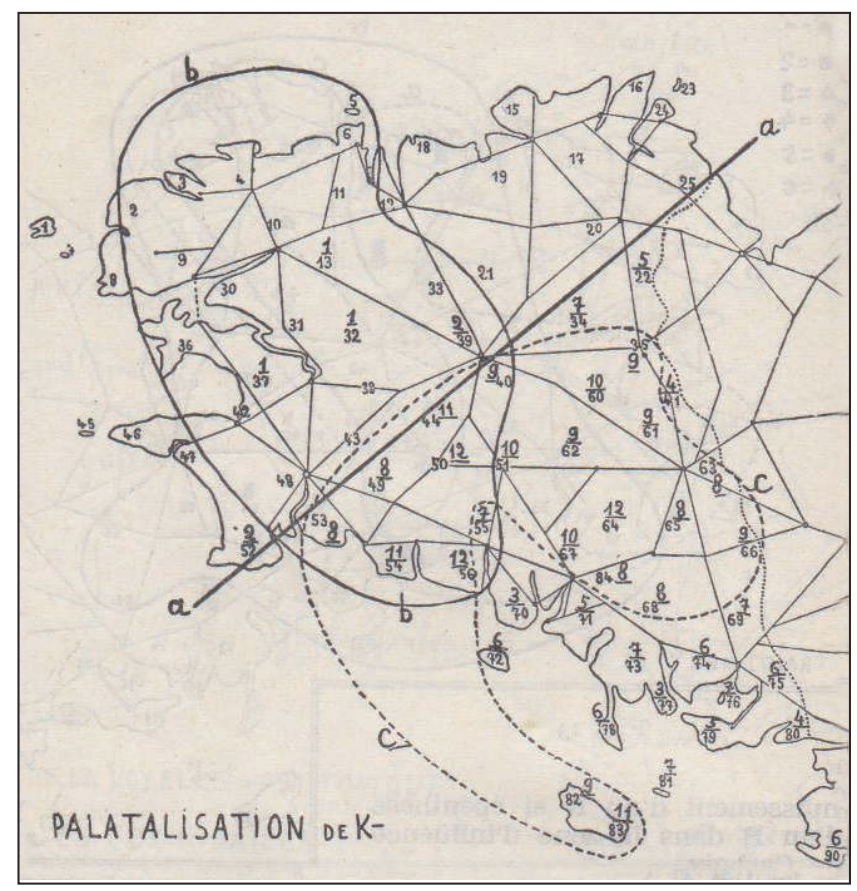

Figure 3 : palatalisation de k selon l'ALBB (Falc'hun (1981 : 18)

Guylaine BT regrette cependant de ne pas pouvoir modifier dans ce module le statut des caractères non reconnus ou mal reconnus. Le Nouvel Atlas Linguistique de la BasseBretagne a noté en exposant certains signes phonétiques pour marquer la faiblesse de leur prononciation : ces derniers ont été assimilés dans Gabmap à des diacritiques modificateurs et sont donc accolés au signe qui précède et non pas traités à part ... heureusement, leur occurrence est trop faible pour perturber les statistiques.

Le second outil du module "Data Inspection », " Distribution maps », permet de visualiser la distribution géographique de chaque forme (lexicale ou phonétique) répertoriée dans les cartes. Du coup, Jean trouve qu'il est dommage qu'on ne puisse pas générer, notamment dans le cas des cartes lexicales, une carte à aires différenciées en couleur montrant la répartition des lemmes ${ }^{6}$.

6. On peut le faire aussi en intégrant une seule carte et en utilisant le module «Cluster analysis», mais on est alors limité à 12 zones maximum ... 
140

\section{Au cœur de la machine dialectométrique}

\subsection{L'algorithme de Levenshtein}

Jean qui avait vu l'algorithme de Levenshtein fonctionner sur un échantillon de données bretonnes (Brun-Trigaud et al. 2015) n'est pas trop perdu pour le moment et l'usage du module « Measuring technique » permettant de vérifier les alignements créés lui a permis d'en comprendre mieux le fonctionnement et de constater que c'est avec une langue comme le breton que l'on éprouve aussi les limites de cet algorithme...

Dans certaines zones, une voyelle inaccentuée tombe, entraînant ainsi la disparition d'une syllabe : ainsi bara «pain » peut parfois devenir bar. Ce type de changement est courant en français (sèchement vs sèch'ment), et que dire du portugais cotinental où le verbe telefonar « téléphoner » peut se réduire à une seule syllabe !

Ainsi pour les mots désignant «le beurre », les relevés des points 16 (Ploudaniel) et 177 (Saint-Philibert), se traduisent dans l'alignement proposé par Gabmap par deux solutions (2), (3):

(2) $\quad 16$

$\begin{array}{lllllllll}16 & & & \mathrm{a} & \mathrm{1} & \mathrm{m} & \tilde{\mathrm{a}} & \mathrm{n} & \\ 177 & \text { ə } & \mathrm{m} & \text { ə } & \text { 1 } & \mathrm{n} & \mathrm{e} & \mathrm{n} & \\ \text { coût } & 1 & 1 & 1 & 0 & 1 & 1 & 0 & 5\end{array}$

(3)

$\begin{array}{lcccccccc}16 & \mathrm{a} & & & \mathrm{1} & \mathrm{m} & \tilde{\mathrm{a}} & \mathrm{n} & \\ 177 & \partial & \mathrm{m} & \partial & 1 & \mathrm{n} & \mathrm{e} & \mathrm{n} & \\ \text { coût } & 1 & 1 & 1 & 0 & 1 & 1 & 0 & 5\end{array}$

Alors que l'interprétation est (4):

(4)

$\begin{array}{lllllllllll}16 & \mathrm{a} & \text { 1 } & \mathrm{m} & \tilde{\mathrm{a}} & & \mathrm{n} & & & \\ 177 & \text { 2 } & & \mathrm{m} & \text { ə } & \text { 1 } & \mathrm{n} & \mathrm{e} & \mathrm{n} & \\ \text { coût } & 1 & 1 & 0 & 1 & 1 & 0 & 1 & 1 & 6\end{array}$

Il est vrai qu'au vannetais amanenn correspond le breton du Nord amann, avec disparition de la dernière syllabe, avec toutefois conservation de l'accent final, ce qui est une anomalie dans les aires à accent d’intensité pénultième.

\subsection{Premières approches dans la mesure des distances linguistiques}

Jusque là, Jean trouvait les choses relativement familières et même envisageait volontiers des applications très pratiques. Mais les choses ont commencé à se corser avec le module « Differences».

Pourtant, grâce à l'outil « Statistics and differences maps », Guylaine a pu lui montrer qu'on pouvait déjà visualiser les distances linguistiques interponctuelles (c'est-à-dire entre les segments reliant les points deux à deux) et que, comme le laissent penser les couleurs choisies de la carte à rayons, plus elles sont intenses, plus les points sont proches linguistiquement et inversement. 
À ce stade déjà on pouvait remarquer une nette différence de répartition entre le corpus basé sur la phonétique et les corpus basés sur le lexique, qui eux ne présentaient aucune divergence.
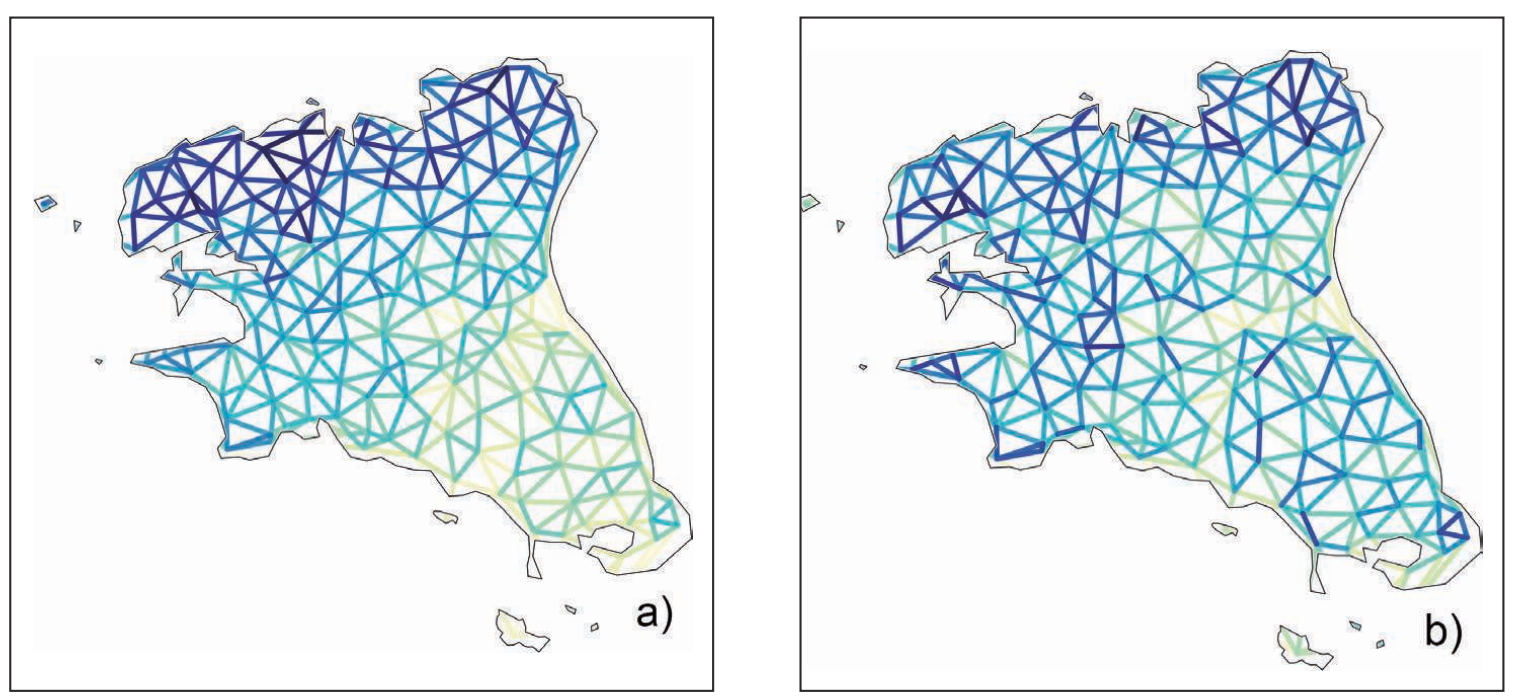

Figure 4 : cartes à rayons a) phonétique; b) lexique (142) et (196)

Dans la carte $4 \mathrm{a}$ (phonétique), on constate qu'il y a plus d'affinités entre les parlers du Nord qu'entre les parlers vannetais plus hétérogènes, tandis que la tendance s'atténue pour le lexique (4b) qui, certes, montre une rupture entre les parlers vannetais et le reste des parlers bretons, mais qui affiche une certaine homogénéité interne à l'intérieur des deux groupes.

\subsection{La répartition des similarités par rapport à un point de référence}

Avec le module « Reference point maps » qui permet, par un dégradé de tons, de visualiser les distances linguistiques entre un point donné et tous les autres points du domaine, Jean y avait retrouvé une notion familière qu'il avait vue à plusieurs reprises dans les travaux de H. Goebl ainsi que dans le travail de G. German.
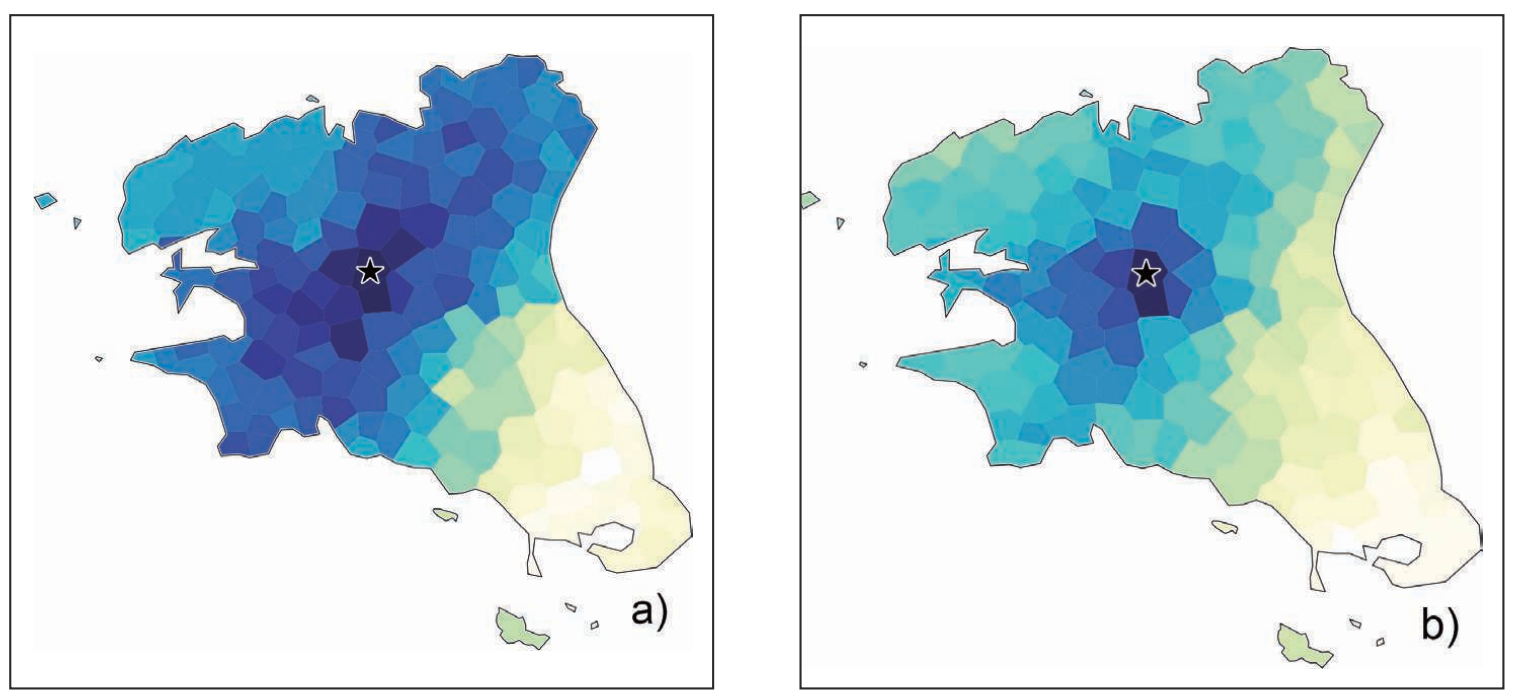

Figure 5 : référence par rapport au pt 88 a) phonétique; b) lexique (142) et (196) 
Si l'on prend, par exemple, un point situé au centre du domaine de NALBB, le point 88 (Collorec), on constate en phonétique l'existence d'une tripartition dialectale du breton avec l'apparition d'une aire centrale intermédiaire entre les deux zones NO et SE observée plus haut. Cette zone est centrée sur Carhaix (l'ancienne Vorgium) où se rencontraient les anciennes voies romaines se rejoignant dans cette ville comme au milieu d'une toile d'araignée (Figure 6). La diffusion semble moins grande du point de vue lexical.

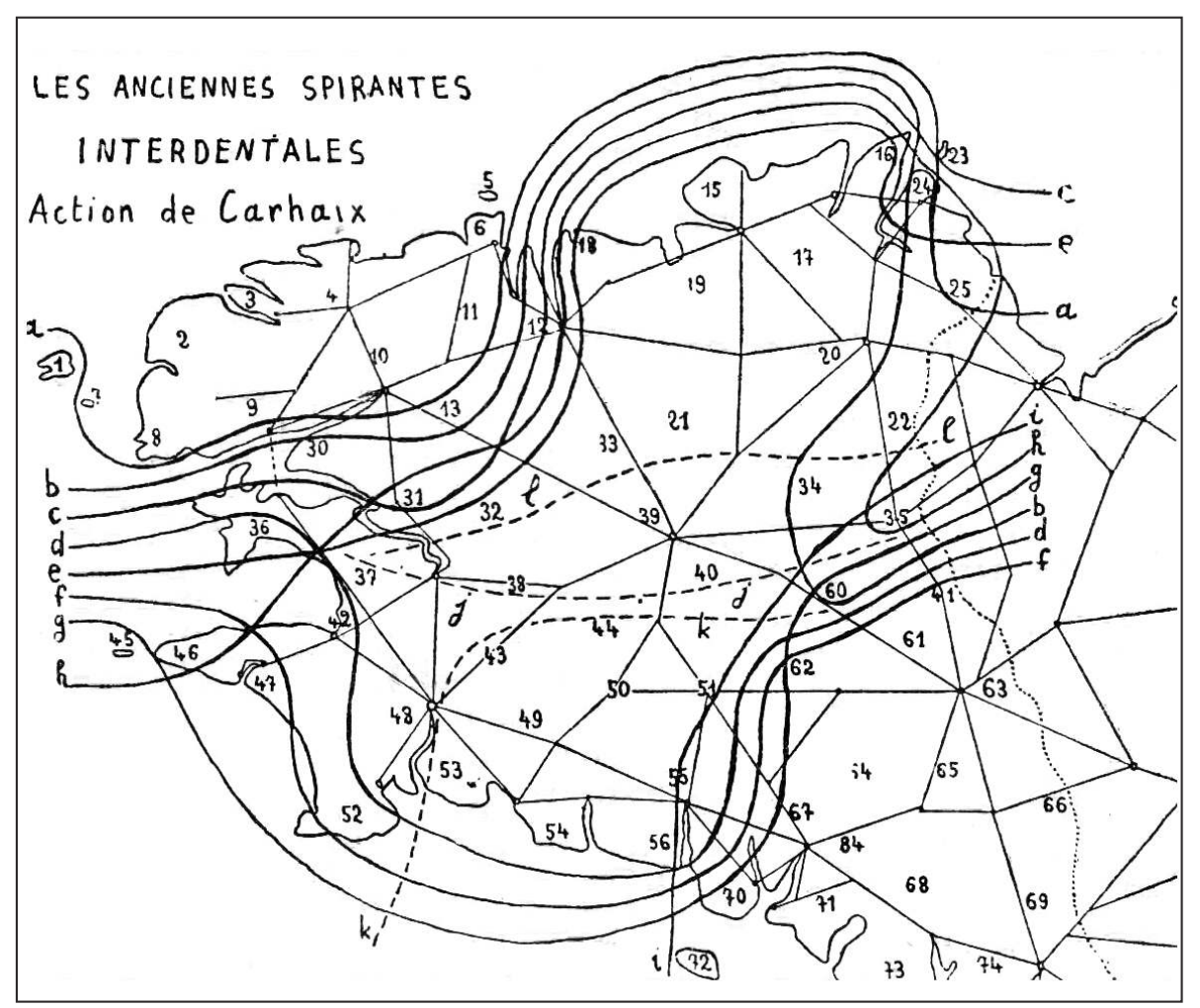

Figure 6 : les anciennes spirantes interdentales (Falc'hun 1981: 140)

\subsection{MDS ...}

Jusque là, tout allait bien, Jean suivait ... mais lorsque nous avons abordé les modules basés sur la discrimination classificatoire, les choses se sont corsées ... Multidimensional Scaling (MDS) petra eo ze ? ou qu'es aco ? Guylaine, qui s'était documentée sur la question, a dû expliquer à Jean que MDS a été introduit en dialectologie par Embleton, 1987 et que cette fonction est utilisée pour montrer le continuum dialectal. Contrairement aux cartes de points de référence (cf. ci-dessus) qui se limitent à montrer les distances linguistiques à partir d'un point vers tous les autres points, MDS permet de visualiser les relations linguistiques entre tous les points dans l'ensemble des données. Chaque point ayant une couleur spécifique, les points semblables du point de vue linguistique ont des couleurs proches. Ceci peut s'illustrer de deux façons : soit par une projection géographique sur la carte avec des couleurs différenciées (Figure 7), soit par un schéma regroupant les points par affinité linguistique sans tenir compte de la répartition géographique (Figure 8). 

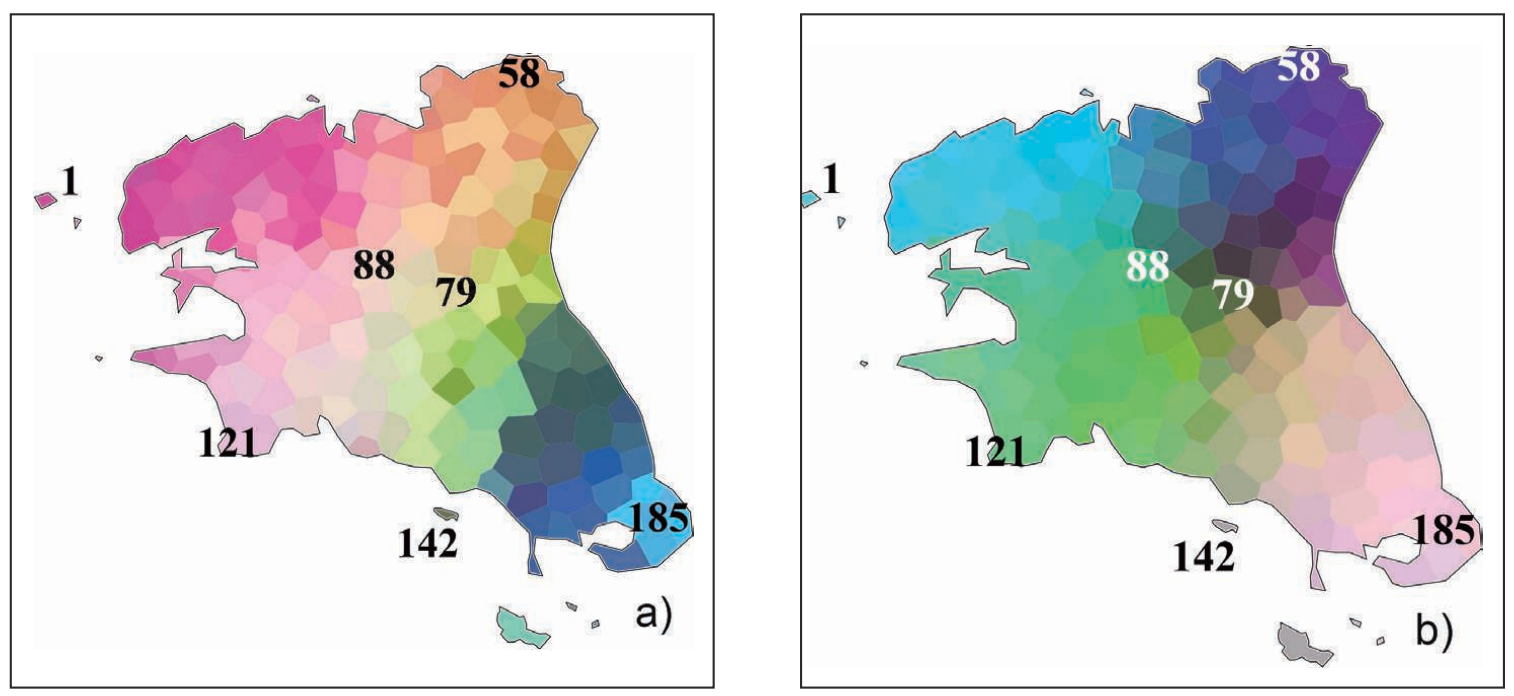

Figure 7 : MDS (3D) a) phonétique ; b) lexique (142) et (196)
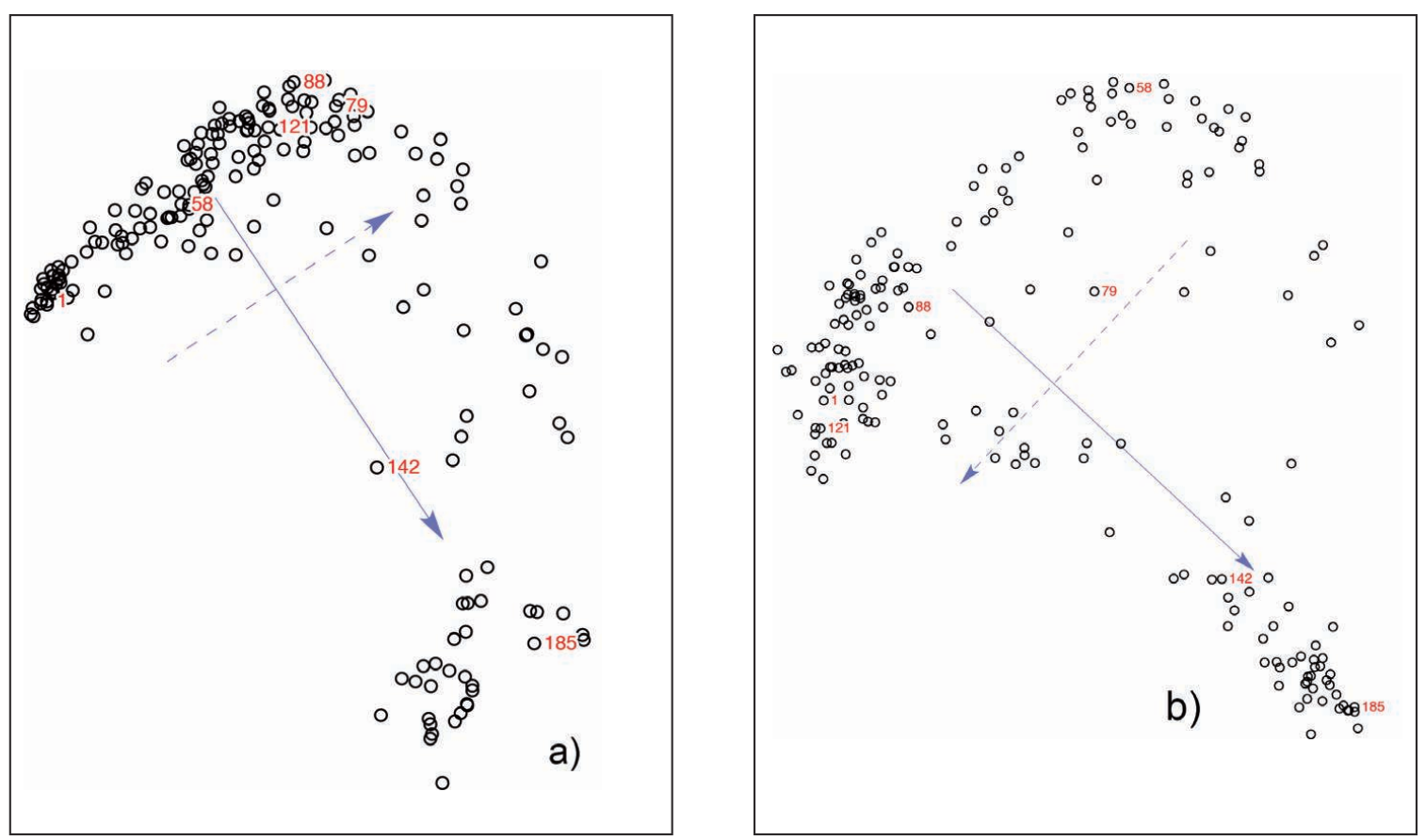

Figure 8 : MDS (2D) a) phonétique ; b) lexique (142) et (196)

Le profil de répartition des points dans les illustrations en 2D est relativement différent pour les deux corpus (où une fois encore les deux corpus de lexique sont identiques). On constate un net regroupement en ce qui concerne la phonétique qui se traduit sur la projection 3D par une vaste zone dans les tons rose à ocre s'opposant à une zone vannetaise dans les tons bleu et vert, tandis que pour le lexique, la répartition est plutôt sur un profil est/ouest où le Léon et la Cornouaille sont un un dégradé du bleu au vert, tandis que le Trégor et le Vannetais sont un dégradé violet à rose.

Il en ressort que si les points 1 (Ile d'Ouessant), 142 (Île-de-Groix) et 185 (Theix) restent 
144

dans la même proportion d'éloignement linguistique, on constate que 58 (Plougrescant), 79 (Paule), 88 (Collorec) et 121 (Penmarc'h) occupent une place très différente dans les deux schémas, ce qui induit que les relations linguistiques sont divergentes selon les corpus.

\subsection{Lanalyse en groupes (Cluster analysis)}

Lanalyse en groupes a également demandé quelques éclaircissements ${ }^{7}$. Si donc MDS montre plutôt les affinités entre les différentes aires, au contraire l'analyse en groupes minimise la distance entre les points et maximise la distance entre les groupes pour faire émerger des frontières plus nettes. À l'inverse de MDS, les couleurs dans Gabmap sont arbitraires et des couleurs proches ne dénotent pas nécessairement une proximité linguistique.

Le principe de l'analyse en groupes est basé sur les algorithmes de classification ascendante hiérarchique (CAH) qui opèrent, chacun à sa façon, des agglomérations successives de $n$ éléments de la matrice de similarité pour aboutir à un arbre de classification clairement structuré (dendrogramme). Il existe de nombreux algorithmes de CAH qui peuvent donner des résultats très différents en fonction de la nature des données (Prokić \& Nerbonne 2008). Les concepteurs de Gabmap en ont retenu quatre: Complete Link, Group Average, Weighted Average et Ward's Method.

Schématiquement, le Complete Link, « lien complet », aussi appelé Furthest Neighbour, le « voisin le plus éloigné », part des paires d'objets à la fois les plus éloignés et les plus semblables en les fusionnant deux à deux au cours d'un processus répétitif.

Group Average, la « Moyenne de Groupe », (ou la « Méthode de Groupe par Paires non pondérées » utilisant des Moyennes Arithmétiques, UPGMA) appartient à un groupe de méthodes de regroupements moyens. Dans la « Moyenne de Groupe », la distance entre deux groupes, quels qu'ils soient, est la moyenne des distances entre les membres des deux groupes en question. La moyenne est pondérée naturellement, en fonction de sa taille. En conséquence, les groupes les plus petits seront moins pondérés et les plus grands le seront plus.

Weighted Average, « Moyenne Pondérée » (ou la Méthode de Groupe de Paire Pondérée utilisant des Moyennes Arithmétiques, WPGMA), exactement comme la « Moyenne de Groupe ", calcule la distance entre les deux groupes comme la moyenne des distances entre tous les membres de deux groupes. Mais dans la « Moyenne Pondérée », les groupes fusionnés ont un poids égal indépendamment du nombre des membres de chaque groupe.

Ward's Method: cette méthode est aussi connue comme la " méthode de désaccord minimal ». À chaque étape de l'analyse, les groupes qui s'amalgament sont ceux qui aboutissent à la plus petite augmentation du carré de la somme des distances de chaque élément à partir de la moyenne de son groupe. Cette méthode utilise une approche d'analyse des variations pour calculer les distances entre les groupes. Elle tend à créer des groupes de même taille.

À titre d'illustration, la figure 9 montre le traitement des données du corpus phonétique

7. Alors que d'autres modules de Gabmap sont bien expliqués et documentés dans l'aide, on aurait souhaité ici avoir des illustrations concrètes pour faire un choix d'algorithme en toute connaissance de cause. 
en 7 groupes au travers des quatre algorithmes:
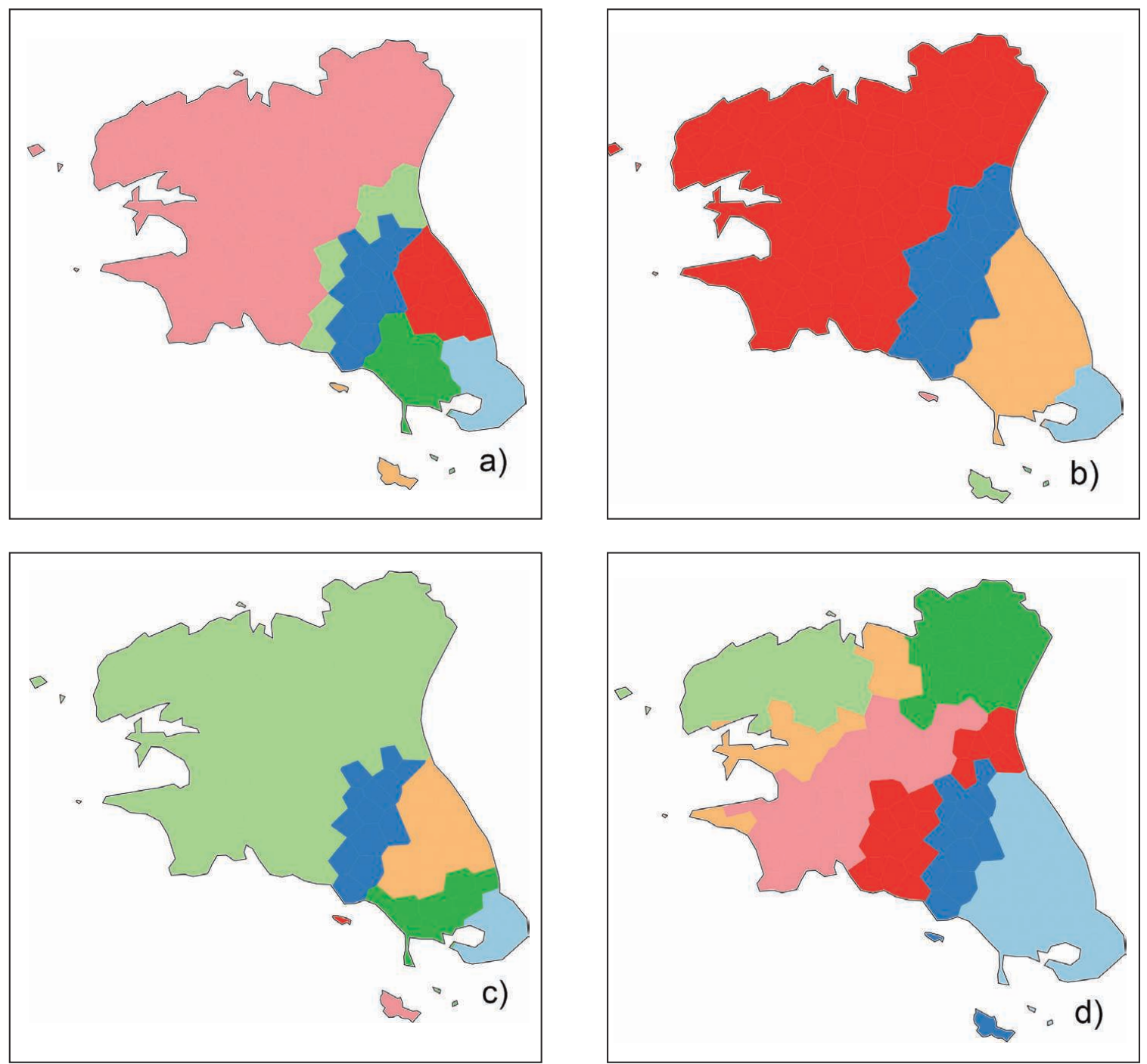

Figure 9 : analyse en 7 groupes sur le corpus de phonétique

a) Complete Link; b) Group Average ; c) Weighted Average ; d) Ward's Method

On remarque bien que les trois premiers algorithmes ne font ressortir que la zone vannetaise au sens large et que seul le quatrième, Ward's Method, propose des aires plus équilibrées. C'est l'un des algorithmes les plus utilisés en dialectologie, et c'est celui-là que nous avons retenu pour quelques exemples d'analyses en groupes, comparativement sur les trois corpus (1. phonétique, 153 cartes ; 2 . lexique, 196 cartes dont $25 \%$ de cartes unilemmes ; 3. lexique, 142 cartes sans cartes unilemmes). 

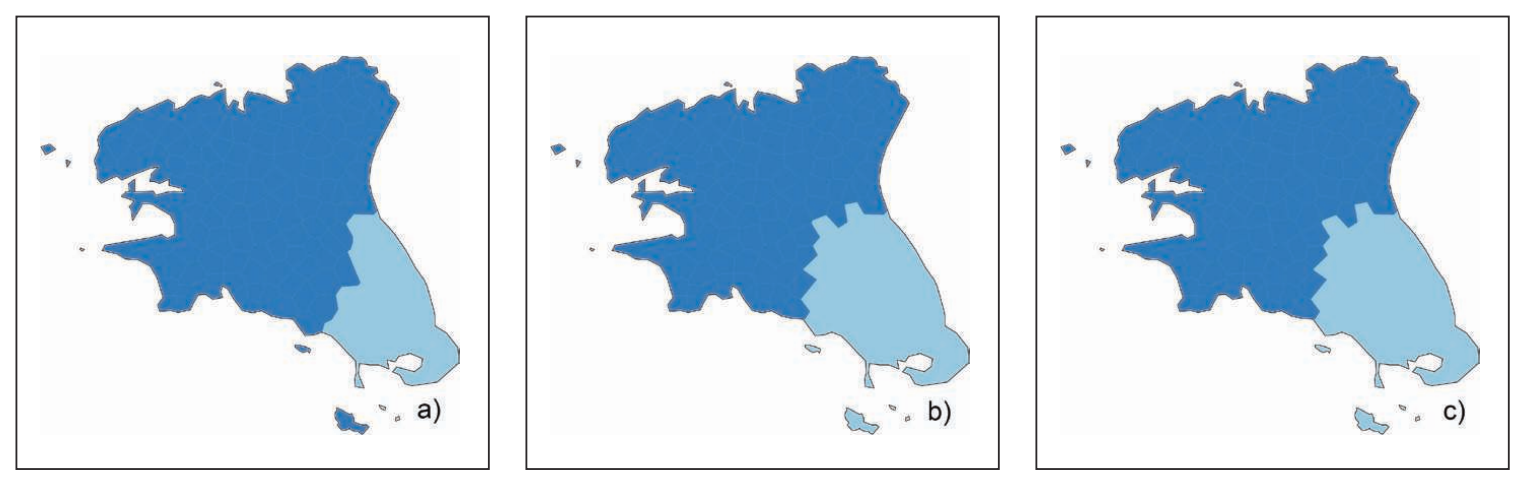

Figure 10 : analyse en 2 groupes, algorithme Ward's Method

a) phonétique ; b) lexique (142) ; c) lexique (196)

Ces cartes (Figure 10) font ressortir au SE l'aire vannetaise, partie bretonnante de l'ancien diocèse de Vannes. Au XVIIe siècle, une langue écrite fut élaborée par les jésuites de Vannes sur la base des parlers de cette zone. Elle dispose d'une graphie, de dictionnaires, de grammaires et d'une littérature spécifiques, tandis que des traductions ont été faites de ce standard vers celui du breton commun au reste de la Basse-Bretagne. La réforme de 1941 a créé une norme dite peurunvan (superunifié) ayant pour objet de fondre les formes écrites de ces deux standards afin de doter la langue d'une graphie unique.

Les aires lexicales sont plus étendues que les aires phonétiques: Falc'hun (figure 11) a pu montrer que des mots français entrent par le SE pour s'étendre ensuite en direction du NO.

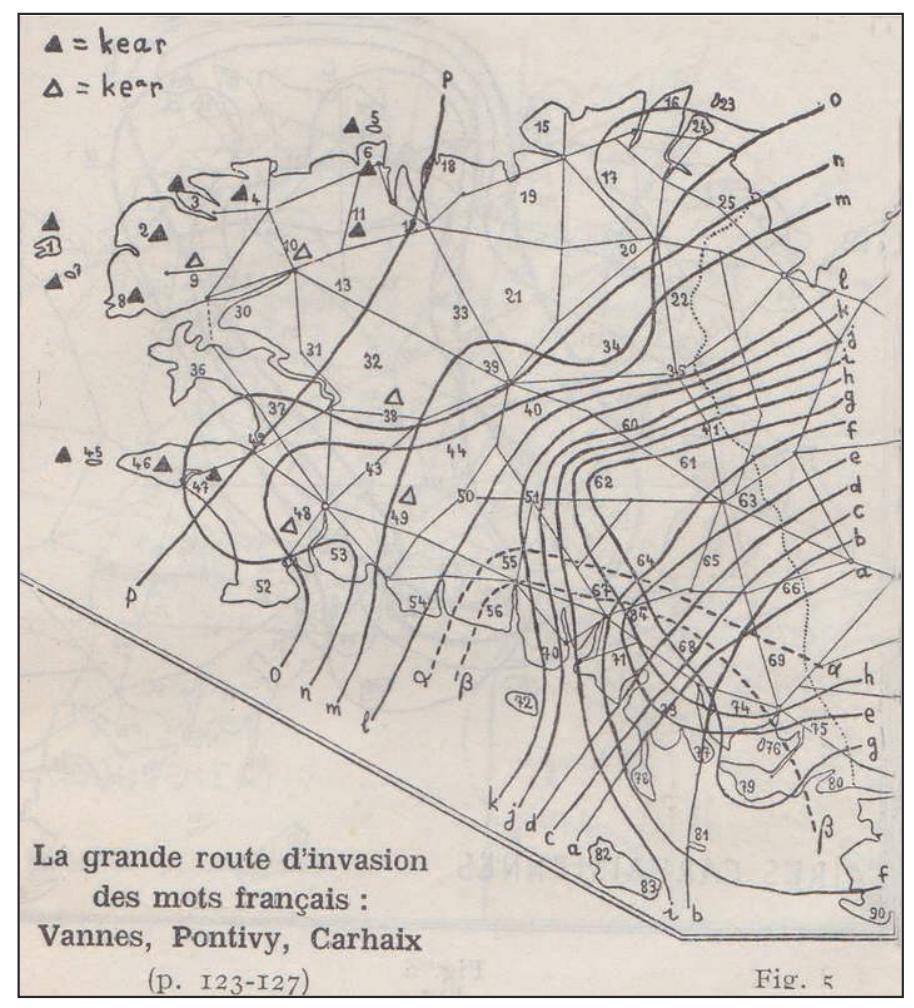

Figure 11 : la grande route d'invasion des mots français (Falc'hun (1981 : 142) 

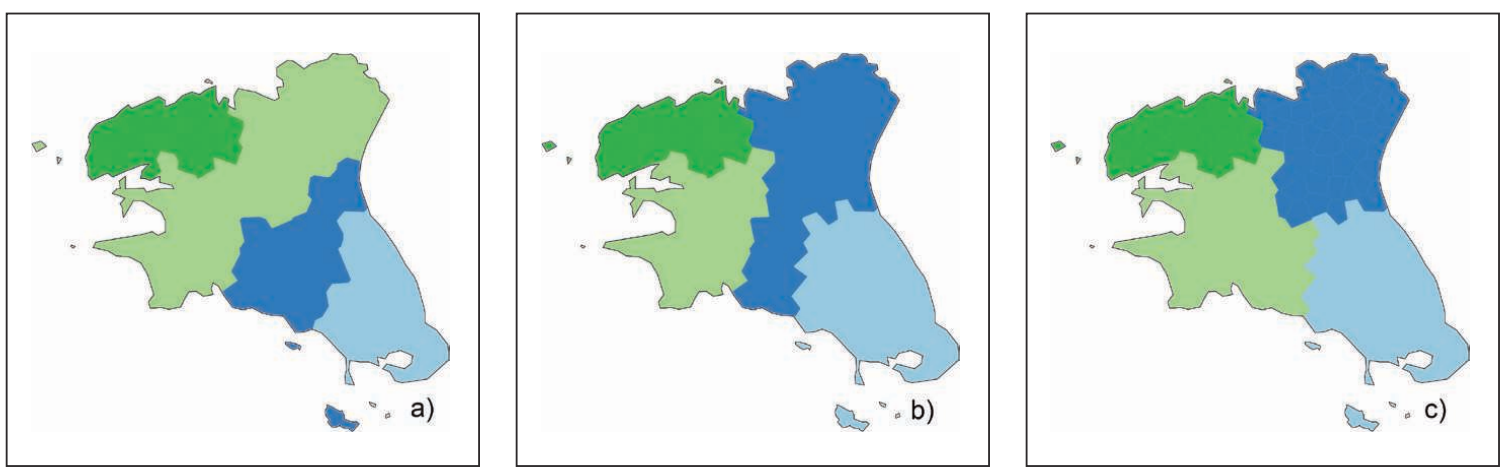

Figure 12 : analyse en 4 groupes Ward's Method

a) phonétique ; b) lexique (142) ; c) lexique (196)

La zone vannetaise est toujours aussi bien délimitée (Figure 12). De ces trois cartes ressort aussi clairement, tant du point de vue lexical que phonétique, la zone remarquablement homogène du NO qui représente une grande partie de l'ancien diocèse de Léon. La rivière de Morlaix marque une coupure nette avec la zone trégorroise à l'est, tandis que les Monts d'Arrée constituent la limite méridionale de la région. C'est sur la base des parlers de cette zone que s'est formé le standard breton ecclésiastique souvent désigné par le sigle KLT (du nom breton des diocèses d'ancien régime Kemper, Leon, Treger). Ici encore, ce sont les jésuites (installés à Quimper parce que le Léon avait refusé de les accueillir) qui en ont établi les règles grammaticales et la graphie, en particulier le fameux trigraphe c'h : comme ils n'étaient en général pas bretonnants, ils avaient imaginé cet artifice afin d'éviter la confusion entre les sons [h] et [S] jusque là écrits tous deux ch. Ainsi, le nom de famille Falc'hun se prononce [falhun] en Léon, tandis que le mot chadenn " chaîne » se lit [Saden]. Il est amusant de constater que cet artifice de néo-bretonnants passe désormais pour une marque de « celtitude »!

La carte phonétique (12a) fait ressortir la tripartition dialectale déjà montrée par Falc'hun, le léonais et le vannetais aux deux extrémités (NO et SE), et une aire oblique, ici divisée en deux, l'une proche des parlers vannetais et l'autre des parlers léonais. Cette zone correspond bien aux observations spontanées des locuteurs sur la relative proximité des parlers centraux. Ainsi, pour le mot « journée » on a en léonais une forme dervez, en vannetais une forme deùeh, et entre les deux devez qui ressemble à un compromis enre les formes extrêmes.

Pour le lexique, la zone trégorroise se détache fort bien dans la carte lexicale (12c), prolongée vers le sud dans la carte lexicale (12b). Il est surprenant de constater que la carte 12c présente une Basse-Bretagne partagée en 4 aires qui correspondent grosso modo aux anciens diocèses. 

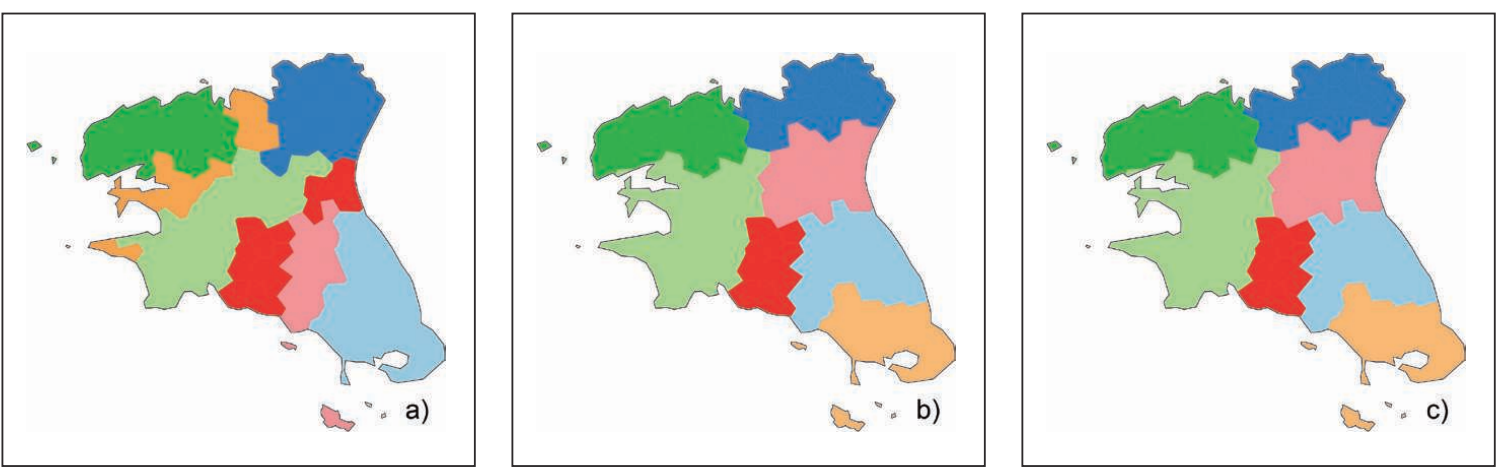

Figure 13 : analyse en 7 groupes Ward's Method

a) phonétique ; b) lexique (142) ; c) lexique (196)

La carte basée sur la phonétique (13a) fait ressortir une zone en orange intermédiaire entre le Léon et les autres zones : le Trégor occidental à l'est de Morlaix, la presqu'île de Crozon, la vallée de l'Élorn et la pointe extrême du cap Sizun. En rouge, la Haute-Cornouaille se distingue en phonétique mais pas du point de vue lexical. La zone située entre Quimper et Quimperlé offre des prononciations originales absentes ailleurs ainsi que des formes lexicales originales. Du point de vue phonétique, le logiciel distingue une aire intermédiaire absente des cartes b et c, qui en revanche font apparaître une aire en beige clair qui correspond à ce qu'on appelle le bas-vannetais, qui contient probablement plus d'emprunts que le reste du vannetais.

\subsection{La carte de groupes flous (Fuzzy Cluster Map):}

Enfin parmi les nombreux outils d'analyse, nous avons choisi de terminer cette aventure dialectométrique par un outil de synthèse qui permet de mettre en avant les aires linguistiques tout en préservant l'aspect des zones servant de transition.
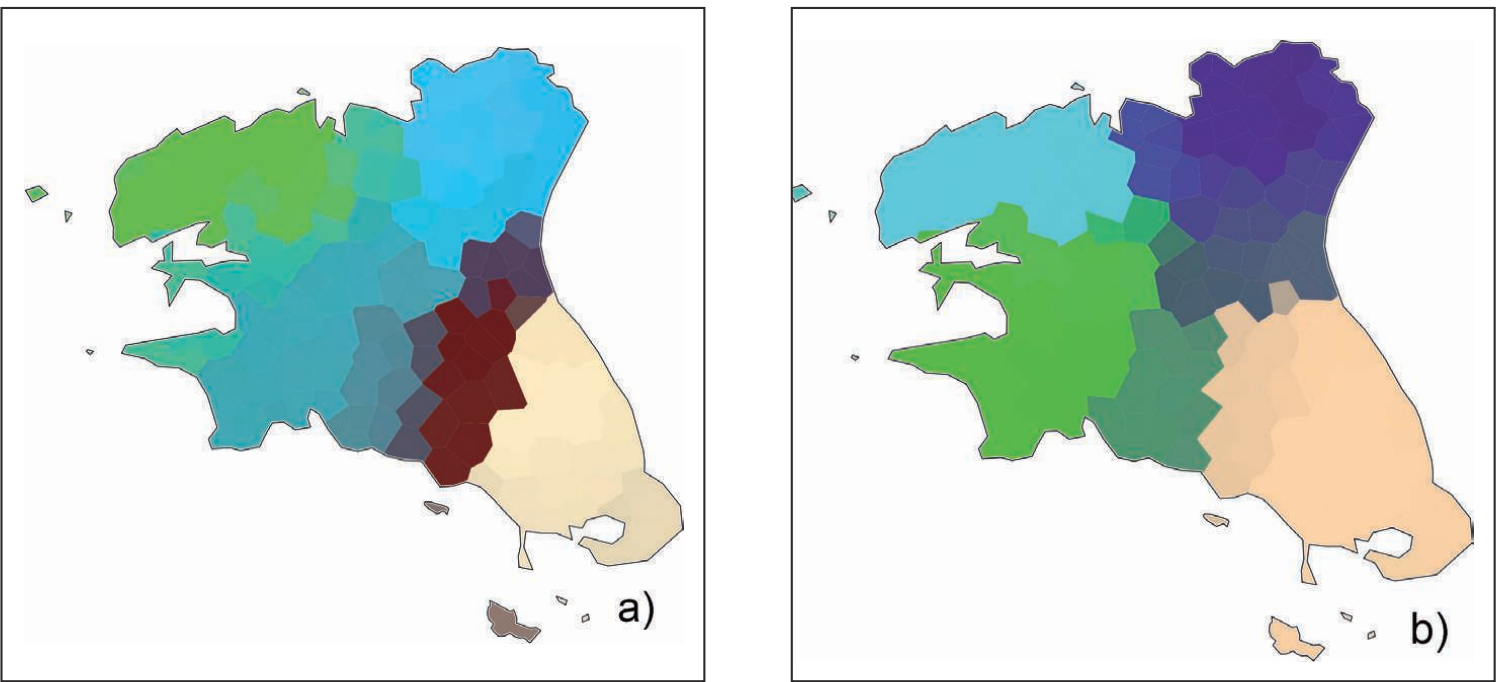

Figure 14 : cartes de groupes flous a) phonétique ; b) lexique (142) et (196) 
La carte phonétique fait ressortir les trois zones, en insistant sur l'aire transitoire entre vannetais et KLT. Jean remarque que ces deux cartes révèlent une certaine unité linguistique des anciens diocèses rappelant la division traditionnelle du breton en quatre dialectes distincts. Ceci est vrai principalement pour le vannetais, le Léon et le Trégor : la Cornouaille historique, qui poussait une pointe jusqu'au centre de la frontière linguistique, se limite au quart SO de la Basse-Bretagne. L'opposition entre ce portrait quadripartite et l'image d'un découpage tripartite des dialectes semblent être en opposition : mais les dialectes existent-ils autrement que dans l'imagination des locuteurs et peuvent-ils être déduits de faits purement linguistiques? Aucune discipline ne peut prétendre détenir la vérité ultime, et il est évident qu'il convient de faire appel à l'histoire, à l'économie et à la sociolinguistique si on veut comprendre la distribution spatiale révélée par la dialectométrie.

\section{Conclusions}

Au terme de nos aventures au pays de la dialectométrie, nous tenons à féliciter les concepteurs de l'application Gabmap qui offre une grande facilité d'utilisation et un nombre importants d'outils mettant la dialectométrie à la portée du plus grand nombre, même si nous n’avons donné qu'un très petit aperçu des possibilités.

Jean LD y a retrouvé les grandes divisions linguistiques qui lui étaient familières, mais il a aussi noté des faits intéressants concernant la répartition des aires dialectales en fonction de la phonétique et du lexique qui ne distribuent pas les faits tout à fait de la même façon. Il semble que la phonétique permette de remonter à une histoire très ancienne, tandis que le lexique, lui aussi porteur d'une très longue histoire, permet dans son évolution de retrouver des faits historiques qui n'ont laissé aucune trace documentaire.

Nous en saurons sûrement davantage à l'issue de la thèse actuellement en préparation de Tanguy Solliec (2014-) qui travaille également à partir du NALBB et qui a pour but de confronter les différentes écoles de dialectométrie sur le domaine du breton. 


\section{Références}

Brun-Trigaud, Guylaine. 2014. Un usage particulier de l'algorithme de Damerau-Levenshtein dans le domaine occitan. In F. Diémoz \& D. Aquino-Weber (eds.), Toujours langue varie ... Mélanges de linguistique historique du français et de dialectologie galloromane offerts à M. le Professeur Andres Kristol par ses collègues et anciens élèves, 127-147. Genève: Droz.

Brun-Trigaud, Guylaine, Jean Le Dû \& Tanguy Solliec. 2015. A new dialectometric approach of the Breton language. In Marie-Hélène Côté, Remco Knooihuizen \& John Nerbonne (eds), The Future of Dialects (Actes du colloque Methods in dialectology XV, Groningen 2014), à paraître.

Costaouec, Denis. 1998. Le breton parlé à La Forêt-Fouesnant (Finistère). Pratique actuelle, problèmes de phonologie et de syntaxe. Paris: Université Paris 5. Thèse (n.p.).

Costaouec, Denis. 2012. Linguistic geography of Breton and sociocultural motivations. STUF - Language Typology and Universals 65(1), 47-64.

Embleton, Sheila. 1987. Multidimensional Scaling as a Dialectometrical Technique. In R. M. Babitch (ed.), Papers from the Eleventh Annual Meeting of the Atlantic Provinces Linguistic Association, 33-49. Centre Universitaire de Shippagan, New Brunswick.

Falc'hun, François. 1981. Perspectives nouvelles sur l'histoire de la langue bretonne. Paris: UGC.

German, Gary. 1984. Une étude linguistique sur le breton de Saint-Yvi. Brest: UBO. Thèse (n.p.).

German, Gary. 1991. Une méthode dialectométrique (assistée par ordinateur) pour l'analyse des atlas linguistiques. La Bretagne linguistique 7. 177-213.

Goebl, Hans \& Pavel Smečka. 2014. Lanalyse dialectométrique des cartes de la série B de l'ALF. Revue de linguistique romane (78). 439-497

Goebl, Hans. 2012. Introduction aux problèmes et méthodes de l'« École dialectométrique de Salzbourg » (avec des exemples gallo-, italo- et ibéroromans). In X. A. Álvarez Pérez, E. Carrilho \& C. Magro (eds.). Proceedings of the International Symposium on Limits and Areas in Dialectology (LimiAr). Lisbon 2011, 117-166. Lisbon: Centro de Linguística da Universidade de Lisboa.

Guerrab, Said. 2014. Analyse dialectométrique des parlers berbères de Kabylie. Paris: INALCO. Thèse. https://tel.archives-ouvertes.fr/tel-01089911.

Guiter, Henri. 1973. Atlas et frontières linguistiques. In G. Straka \& P. Gardette (eds.), Les dialectes romans de la France à la lumière des atlas régionaux, 61-109. Paris: éditions du CNRS.

Heeringa, Wilbert. 2004. Measuring dialect pronunciation differences using Levenshtein distance. Groningen: University of Groningen, Ph.D. dissertation.

Kellerhals, Sandra. 2014. Dialektometrische Analyse und Visualisierung von schweizerdeutschen Dialekten auf verschiedenen linguistischen Ebenen. Zürich: Universität, Geographisches Institut. Mémoire de Master. http://www.geo.uzh.ch/en/ units/giscience-gis/research/msc-theses/fontsize/0

Le Dû, Jean. 2001. Nouvel Atlas Linguistique de la Basse-Bretagne. Brest: CRBC. 2 volumes. 187 points d'enquête, 600 cartes.

Le Roux, Pierre. 1924-1963. Atlas linguistique de Basse-Bretagne. Rennes \& Paris: Plihon- 
Hommay. 6 volumes. 77 points d'enquête. 600 cartes.

Nerbonne, J., R. Colen, C. Gooskens, P. Kleiweg \& T. Leinonen. 2011. Gabmap -- a web application for dialectology. Dialectologia: revista electrònica. 65-89.

Nerbonne, John \& Wilbert Heeringa. 2001. Computational Comparison and Classification of Dialects. Dialectologia et Geolinguistica (9). 69-83.

Nerbonne, John \& Wilbert Heeringa. 2010. Measuring dialect differences. In J.-E. Schmidt \& P. Auer (eds.). Language and Space: Theories and Methods, 550-567. (Handbooks of Linguistics and Communication Science). Berlin: Mouton de Gruyter.

Prokić, Jelena \& John Nerbonne. 2008. Recognize Groups among Dialects. In John Nerbonne, Charlotte Gooskens, Sebastian Kürschner \& Renée van Bezooijen (eds), International Journal of Humanities and Arts Computing, Special Issue on Language Variation. 153-172.

Séguy, Jean. 1971. La relation entre la distance spatiale et la distance lexicale. Revue de Linguistique Romane (35). 335-357.

Séguy, Jean 1973. La dialectométrie dans l'Atlas linguistique de la Gascogne. Revue de Linguistique Romane (37). 1-24.

Snoeck, Conor. 2014. Review of Gabmap: Doing Dialect Analysis on the Web. Language, Documentation \& Conservation (8). 192-208.

Solliec, Tanguy. 2014- . The Dialectometry of the Breton-speaking area : issues, methodologies and application. Quantify and qualify the variation in Breton, an insight into its synchronic dynamics and symbolic geography. Brest: UBO. Thèse.

Wieling, Martjin \& John Nerbonne. 2015. Advances in Dialectiometry. Annual Review in Linguistics 1(1). 243-264. 